\title{
SEGUIMIENTO A ECONOMISTAS EGRESADOS DE LA UNIVERSIDAD DE NARIÑO
}

\section{TRACKING ECONOMISTS GRADUATES FROM THE UNIVERSITY OF NARIÑO}

\section{RASTREAMENTO ECONOMISTAS GRADUADOS UNIVERSITÁRIOS NARIÑO}

\author{
Por: BUESAQUILLO CUAICUAN - William Fernando, PAZ GAMBOA - Rosa María
}

Economista Universidad de Nariño. Diplomado Internacional en Emprendimiento y Gestión de Proyectos para el Desarrollo y la Innovación, Campus Iberus, Gobernación de Nariño, Organización de Estados Iberoamericanos. Email: wfbc87@hotmail.com - Colombia.

Magister en Administración y Especialista en Gerencia de proyectos Universidad Regional Autónoma de Los Andes. Contador Público Corporación Universitaria de Ciencia y Desarrollo. Administrador de Empresas Universidad Nacional Abierta y a Distancia. Docente Universitario, Par Académico Ministerio de Educación Nacional. E-mail: rpaz@umariana.edu.co - Colombia.

\section{RESUMEN}

El presente artículo corresponde a los resultados obtenidos en la investigación denominada "Propuesta para la creación de un programa de seguimiento a economistas egresados de la Universidad de Nariño 2008-2013". Los objetivos establecidos se derivan del propósito de conocer: situación socioeconómica, trayectoria educativa y percepción del profesional en temas referentes al programa de Economía. Por último se desarrolla la propuesta de seguimiento a egresados contemplada como un indicador de la calidad universitaria.

En el desarrollo del estudio se tuvo en cuenta los trabajos de grado "Caracterización de los egresados del programa de Economía de la Universidad de Nariño" y "Caracterización y aporte al desarrollo regional de los economistas egresados del programa de Economía de la Universidad de Nariño sede Pasto, 2010" elaborado por los economistas Robert Wilson Ortiz López y Pablo Sebastián Martínez Solarte, respectivamente, lo que permitió efectuar un análisis comparativo en algunos aspectos de los graduados en el programa de Economía de la Universidad de Nariño.

Palabras clave: seguimiento a egresados, situación socioeconómica, trayectoria educativa, percepción, calidad universitaria.

JEL: 120. 


\begin{abstract}
This article corresponds to the results obtained in the research called "Propuesta para la creación de un programa de seguimiento a economistas egresados de la Universidad de Nariño 2008-2013". The stated objectives are derived from the purpose of knowing: socioeconomic status, educational background and professional perception of issues relating to economics program. Finally the proposed graduate follow-up seen as an indicator of university quality is developed.

In the course of study there were taken into account the works of degree "Caracterización de los egresados del programa de Economía de la Universidad de Nariño" and "Caracterización y aporte al desarrollo regional de los economistas egresados del programa de Economía de la Universidad de Nariño sede Pasto, 2010" prepared by economists Robert Wilson and Sebastián Ortiz Pablo Martínez Lopez Solarte respectively, which allowed a comparative analysis in some aspects of the graduates in Economics program at the University of Nariño.
\end{abstract}

Keywords: graduate follow-up, socioeconomic status, educational path, perception, university quality.

JEL: 120.

\title{
RESUMO
}

Este artigo corresponde aos resultados obtidos na pesquisa chamada"Propuesta para la creación de un programa de seguimiento a economistas egresados de la Universidad de Nariño 2008-2013". Os objetivos declarados são derivadas do propósito de conhecer: condição socioeconômica, percurso educativo e percepção de questões profissionais relativas ao programa de economia. Finalmente, a pós-graduação seguimento proposto visto como um indicador de qualidade da universidade é desenvolvido.

No decorrer do estudo das obras de grau "Caracterización de los egresados del programa de Economía de la Universidad de Nariño" e" caracterización y aporte al desarrollo regional de los economistas egresados del programa de Economía de la Universidad de Nariño sede Pasto, 2010", elaborado pelos economistas Robert Wilson e Sebastián Ortiz Pablo Martínez López Solarte, respectivamente, o que permitiu uma análise comparativa em alguns aspectos do programa de pós-graduação em Economia na Universidade de Nariño.

Palavras-chave: Ensino de acompanhamento, status socioeconômico, percurso educativo, a percepção, a qualidade da universidade.

JEL: 120 


\section{INTRODUCCIÓN}

La calidad educativa determina en cierta medida el grado de desarrollo al que puede aspirar una región. En este aspecto juega un papel preponderante la academia que es la encargada a través de sus facultades y programas, de formar profesionales aptos para el desarrollo económico y social.

El seguimiento a egresados ha permitido que diversas instituciones puedan contar con información fundamental en la formulación de políticas y estrategias pertinentes a las exigencias del mercado. La evaluación periódica del impacto social y laboral del egresado ha fortalecido los procesos de autoevaluación mejorando los programas académicos y la relación universidad-egresados. La medición del impacto en el medio no es una tarea fácil, es necesario que su articulación esté en manos de profesionales idóneos que trabajen en un ambiente de fraternidad, confidencialidad y seguridad, permitiendo la obtención de resultados favorables.

En el presente artículo se tiene como finalidad conocer: la situación socioeconómica, trayectoria educativa y percepción del profesional en temas referentes al programa de Economía, y mediante los resultados encontrados elaborar la propuesta de seguimiento a egresados. Por último, se expresa agradecimientos a todos los egresados encuestados por su disposición y aportes a la investigación. Sin su colaboración los resultados obtenidos no hubiesen sido posibles.

\section{SEGUIMIENTO A EGRESADOS}

La formación profesional influye en el crecimiento y desarrollo de una región, las normas y políticas que rigen en Colombia estipulan la importancia de la educación de la siguiente forma: la Constitución Política de Colombia en los artículos 67 y 69 hace énfasis en la formación educativa como un derecho de las personas y una obligación del Estado. Asimismo, la Ley 30 de 1992 identifica la educación superior como un "proceso dinamizador que facilita el desarrollo de las potencialidades del ser humano de una manera sistémica" (Constitución Nacional Ley 30 de 1992, 1992: 1). La Ley 115 de 1994 expone las normas legales de educación en Colombia reguladoras del servicio público, su contenido se desarrolla teniendo como base los principios de la Constitución Política Nacional en aspectos como el aprendizaje, la investigación y la cátedra en las instituciones existentes en el territorio nacional. La Ley 1188 de 2008 establece las normas mediante las cuales se regula el registro calificado de Programas académicos. Finalmente, el Decreto 1295 de 2010 reglamenta el registro calificado, la oferta y el desarrollo de los diferentes programas de educación superior.

Por otra parte, la medición del impacto del egresado y su evaluación constituyen un factor relevante en los procesos de acreditación, puesto que "resulta indispensable fortalecer las comunidades académicas de disciplinas, profesiones, ocupaciones y oficios, como factor fundamental para alcanzar altos niveles de calidad en los distintos programas. La acreditación es un medio para reconocer hasta dónde se cumple ese proceso satisfactoriamente y para establecer qué tanto la educación 
superior está respondiendo a las exigencias que le plantea el desarrollo del país”. (Consejo Nacional de Acreditación, 2013:7).

Para orientar la evaluación educativa en alta calidad el Consejo Nacional de Acreditación ha delimitado los aspectos a evaluar en 40 características y 10 factores, los cuales se listan así: Misión, Visión y Proyecto Institucional y de Programa, Estudiantes, Profesores, Procesos académicos, Investigación y creación artística y cultural, Visibilidad nacional e internacional, Impacto de los egresados sobre el medio, Bienestar institucional, Organización, administración y gestión, y Recursos físicos y financieros. Para esta investigación se destaca el Impacto de los egresados sobre el medio, analizado a través de la ubicación y las actividades que desarrollan en el ámbito social y académico.

En cuanto a la evaluación permanente se tiene que "la relación entre el título obtenido y el desempeño profesional puede verse afectada no sólo por las variables del mercado laboral, sino también por las variables sociales, familiares y educativas que caracterizan a cada egresado" (Red Graduados, 2006:17). Es imperante que las Universidades, en este caso la Universidad de Nariño incluya en su Plan de Desarrollo, programas orientados al seguimiento y medición del impacto de sus egresados.

La formación profesional requiere de una formación integral para que el egresado sea ético en sus labores cotidianas, fomentando la autoevaluación y cambios en su conducta en pro de su desarrollo individual. Esto fortalecerá su capacidad para la toma de decisiones acertadas y le motivará a ser parte activa de los procesos de desarrollo regional. A esto Luis Aurelio Ordóñez (2013:161) señala: "la clave para alcanzar la senda de desarrollo educativo superior no está tanto en las condiciones económicas y sociales de un país, ni en las reformas puntuales elegidas, sino en la permanencia en el tiempo de un conjunto de valores comunes que hagan de quienes realizan la actividad educativa (estudiantes, profesores, administradores, familias) los mejores evaluadores de su propio desempeño, en procesos en los que todos contribuyan a un propósito público que supere los intereses privados e individuales".

\section{CARACTERIZACIÓN SOCIO-ECONÓMICA DE LOS ECONOMISTAS EGRESADOS DE LA UNIVERSIDAD DE NARIÑO}

El seguimiento a egresados fortalece los procesos académicos de las instituciones de educación superior beneficiando de manera directa e indirecta a la comunidad académica: la universidad adquiere una base de datos que le permite analizar el desempeño e impacto del profesional, el graduado conoce si su formación académica es acorde a los requerimientos del mercado, y el estudiante tendrá la oportunidad de capacitarse de acuerdo a las exigencias de la región.

Al estudiar el periodo 2008-2013 en el Programa de Economía se tiene un total de trescientos cuarenta y dos (342) titulados, de los cuales se encuestó a ciento veintitrés (123) personas, tomando un nivel de confianza del noventa y cinco por ciento (95\%), y un margen de error del siete por ciento (7\%). Esta muestra se distri- 
buyó proporcionalmente al número de graduados en cada año permitiendo conocer cuál fue la situación socioeconómica, la trayectoria educativa, y la percepción del profesional; proporcionando las herramientas necesarias para la propuesta de seguimiento a egresados.

Al analizar los antecedentes de la investigación presentados por Ortiz (2003) y Martínez (2010) se tiene para el primer estudio una población aproximada de mil ochocientos (1800) graduados desde la primera promoción hasta el año 2002, con un grado de confianza del noventa por ciento (90\%) y un margen de error del cinco por ciento (5\%). Se aplicó una encuesta a doscientos treinta y cuatro (234) personas de manera aleatoria, es decir, sin considerar el año de egreso, el género, o ningún otro tipo de estratificación. En el segundo trabajo, con un número aproximado de mil nueve (1009) egresados en el periodo 1979-2008, con un grado de confianza del noventa y cinco por ciento (95\%), y un margen de error del cinco por ciento (5\%), se encuestó a doscientos setenta y ocho (278) economistas mediante un muestreo aleatorio probabilístico y estratificado en un rango de diez años. No obstante, debido a la multiplicidad de objetivos y respuestas encontradas en cada investigación, solo fue posible analizar y profundizar algunas variables del presente estudio.

\section{DATOS GENERALES}

El porcentaje de hombres y mujeres egresados en el programa de Economía desde la primera promoción hasta el periodo 2013 ha sido equitativo, reflejando igualdad de género en oportunidades y posibilidades de vinculación laboral.

En el estado civil para el periodo 2008-2013 se tiene un 74\% de economistas solteros. Al mismo tiempo los egresados en unión libre, casados y separados corresponden el 14,6\%, 9.8\% y 1,6\% respectivamente; indicando la presencia de profesionales con un cierto grado de compromiso. En Martínez (2010) el número de solteros es de 39\%, siendo el de mayor participación. Además, es notoria la cantidad de personas casadas, en unión libre y separadas, representadas en un 35\%, 23\% y $5 \%$ proporcionalmente. Por último, no se encontró población con estado civil de viudez en las tres investigaciones.

Al considerar el grado de responsabilidad, para el periodo $2008-2013$ el 49,6\% no tienen personas a cargo. El 29,2\% tiene una (1) persona, el 16,3\% dos (2) personas y el 4,9\% tres (3) personas. En Martínez (2010) la mitad de los egresados tienen personas a cargo, sin embargo en el periodo 1979-1988 la mayor parte tiene hijos que superan la mayoría de edad, y no dependen económicamente de sus padres.

Con referencia al estrato socioeconómico, se encontró un alto porcentaje de economistas jóvenes en los estratos 2 y 3. Conjuntamente, la participación del estrato 1 y 4 es poco significativa, y en los estratos 5 y 6 no hay profesionales, coincidiendo con los datos de Martínez (2010), especialmente en el periodo 1999-2006. Lo anterior sitúa a los egresados principalmente en los estratos sociales medios, imposibilitando cubrir cómodamente la totalidad de sus necesidades básicas. 
En cuanto a la ubicación de la residencia se obtuvo que el 61\% se encuentra en el municipio de Pasto, un 25\% en otros municipios del departamento de Nariño, y el $12 \%$ y $2 \%$ respectivamente, por fuera del departamento y en el exterior. Con respecto, al lugar de habitación, un $71 \%$ vive en casas de las cuales gran parte son familiares, seguidas de viviendas propias y domicilios arrendados o anticresados. $\mathrm{Al} \mathrm{mismo} \mathrm{tiempo,} \mathrm{se} \mathrm{encuentra} \mathrm{que} \mathrm{un} 27 \%$ de economistas habitan apartamentos, específicamente en calidad de arrendatarios, seguido de apartamentos familiares, y muy pocos gozan de uno propio. Por último, el $2 \%$ habita una finca familiar o un cuarto arrendado. Para Martínez (2010) se tiene que el 46\% arrienda, un 35\% tiene vivienda propia, y el 19\% habita una vivienda familiar. En contraste, se tiene un aumento en el número de egresados que habitan viviendas familiares debido a su juventud y soltería, lo que no conlleva obligaciones de manera inmediata. Es preocupante el alto porcentaje de economistas arrendatarios, si se toma este pago como limitante de otros gastos, tales como alimentación, vestuario y/o recreación, entre otros.

Por último, al referirse a servicios básicos la totalidad de los egresados cuenta con energía eléctrica, el 99\% posee agua, el 97\% acueducto, el 89\% alcantarillado, el 86\% internet y el 70\% servicio telefónico; lo anterior en razón, de que un gran número de profesionales habita en el municipio de Pasto o en municipios aledaños que cuentan con estos servicios.

\section{SITUACIÓN LABORAL}

Al examinar la situación laboral del economista, el 62\% es empleado de tiempo completo, teniendo correspondencia con los datos encontrados en Ortiz (2003) 76\% y Martínez 2010 (54,8\%); asimilando una tendencia a lo largo de los años. El 13\% es sub-empleado, siendo un valor cercano a Martínez (2010) 15,1\%, con relevancia en la población joven y/o recién graduada. En Ortiz 2003 solo el 5\% se encuentra en situación de sub-empleo. Para los egresados independientes no se observa una variación considerable, la cantidad de personas en esta condición oscila entre el $13 \%$ y 18\%; expresando una baja significancia que afecta el SER del economista, concebido como un profesional capaz de crear y transformar el medio social mediante la constitución empresarial y la sostenibilidad ambiental. Por último, el grado de desempleo para Ortiz 2003 fue de 6\% siendo un índice bajo. En Martínez (2010) fue del 14\%, es decir, en el transcurso de estas dos investigaciones se incrementó la deserción laboral en un $8 \%$. No obstante, al analizar simultáneamente las cifras de desempleo encontradas para el periodo 2008-2013 (7\%) y el promedio nacional $11 \%^{1}$ hay una fortaleza por el aumento de economistas vinculados laboralmente en los últimos años.

1. Dato consultado en noviembre del 2013 en Portafolio. Para mayor información visite: http://www. portafolio.co/economia/desempleocolombia. 
En la forma de contratación, los empleados a término fijo representan el 50\%, siendo un número mayor que en Ortiz (2003) 22\% y en Martínez (2010) 26\%, debido a su juventud y baja experiencia. Para el empleado por periodo indefinido, en Ortiz (2003) 54\% se tiene más economistas en esta situación que en Martínez (2010) $33 \%$, y que los encontrados para los años 2008-2013 (41\%), debido a que gozan de mayores conocimientos en su vida laboral. Por último, para el periodo 2008-2013 se tiene que el $6 \%$ está contratado por provisionalidad y el $2 \%$ en periodo de prueba.

Independientemente del tiempo de trabajo, el área de desempeño con mayor participación de economistas es la financiera (37\%), seguida del área de talento humano (20\%), de las áreas comerciales (16\%), de las áreas técnicas o de producción (12\%), y de proyectos (10\%). Conjuntamente, los egresados en empresas dedicadas a actividades de agricultura, ganadería, caza y silvicultura representan el 4.3\%. A lo anterior, sobresale la semejanza existente entre las investigaciones, por la alta cantidad de profesionales vinculados al sector terciario, debido a que muchos residen en ciudades capitales. Por otra parte, el $41 \%$ de los egresados empleados consiguió una vacante por ayuda de terceras personas, el $27 \%$ por concurso de mérito, el $20 \%$ por iniciativa propia y el $12 \%$ restante por invitación.

Para el tipo de empresa, existe semejanza entre los economistas empleados en empresas públicas y en entidades privadas. Al mismo tiempo, el $84 \%$ se encuentra afiliado al régimen contributivo, el $10 \%$ al régimen subsidiado, y el $6 \%$ restante a la medicina pre-pagada. Los resultados indican que sin importar el tipo de afiliación, el profesional puede acceder a los servicios adecuados que le garanticen su recuperación y protección personal.

Ahora bien, al estudiar los ingresos del economista los cuales representan sus condiciones de productividad y capacidad de compra, para el periodo 2008-2013 se encontró que el ingreso del $52 \%$ de los profesionales empleados oscila entre $1 \mathrm{y}$ $2 \mathrm{SMMV}^{2}$, para el $37 \%$ entre 2 y 4 SMMV, y solo el $11 \%$ obtiene ingresos superiores a 4 SMMV. Para Ortiz (2003) el 55\% de los titulados recibió un ingreso que fluctuaba entre los 2 y 5 SMMV, seguido de un $20 \%$ que devengaba entre 1 y 2 SMMV, mientras que un 15\% obtenía más de 5 SMMV. Para Martínez (2010), el 50\% tuvo un ingreso entre 1 y 2 SMMV, el 6\% entre 2 y 5 SMMV, y el 18\% más de 5 SMMV. De acuerdo a las investigaciones en promedio se tiene ingresos entre 1 y 2 SMMV caracterizado principalmente por los economistas recién graduados. Por último, en Ortiz (2003) se tiene los profesionales con los ingresos más altos.

2. Hace referencia al salario mínimo mensual vigente en Colombia el cual varía en las tres investigaciones debido a los procesos inflacionarios y las políticas tomadas por la banca central. No es de olvidar que este incremento salarial es nominal, puesto que las políticas aplicadas en su manejo se realizan para mantener el poder adquisitivo y la capacidad de compra de las personas. A lo anterior, para el periodo 2008-2013 en el momento de la investigación el salario mínimo mensual vigente en moneda colombiana era de \$589.500, para Martínez (2010) correspondió a \$ 515.000 , y en Ortiz (2003) fue de \$332.000. 
$\mathrm{Al}$ analizar la situación de los economistas independientes se observa una baja participación en lo referente a emprendimiento, seguido de un impacto mínimo sobre el entorno regional a saber: en Ortiz (2003) hay un 13\% y en Martínez (2010) un 15\% de trabajadores independientes, destacando el periodo 1989-1998 como el decenio de egresados con mayor iniciativa.

Los economistas independientes en el periodo 2008-2013 representan el 18\% del total de la población. La mayoría ubica sus empresas o negocios en el sector terciario siendo este segmento una fuente de generación de empleo y un mecanismo de sostenibilidad económica. El 32\% de ellos, se dedica a actividades de servicios comunitarios, sociales y/o personales, seguido de la actividad de servicios de salud, y el comercio al por menor con un $18 \%$ cada uno, respectivamente. Las actividades de construcción, administración pública, los hoteles y restaurantes representan un 9\% proporcionalmente, y las actividades empresariales constituyen el 5\% siendo esta última la población menos significativa. Por último, no se encontró trabajadores independientes en el sector primario y secundario.

Con objeto de establecer la reciprocidad entre el promedio mensual de ganancias y el número de empleados, se tiene que los economistas independientes en su mayoría son microempresarios, de los cuales el 73\% no tiene empleados a su cargo; el 18\% tiene a su cargo, entre uno (1) y cinco (5) empleados; el 9\% entre seis (6) y diez (10) empleados. En lo referente a las utilidades, el 59\% obtiene ganancias que oscilan entre 2 y 4 SMMV, el 23\% entre 4 y 10 SMMV, y el 18\% entre 1 y 2 SMMV.

Frente a la seguridad social, el 77\% de los egresados no tiene afiliación a salud, pensión y riesgos laborales, tampoco reciben prestaciones sociales. La razón generalizada, es que a fin de disminuir costos optan por aprovechar los beneficios de vinculación al régimen subsidiado.

Con respecto al apoyo que han recibido los economistas independientes de entidades públicas y/o privadas, el 91\% desconoce estos beneficios. Es evidente la falta de información, la distancia, y/o poco interés en asistir a las capacitaciones que se dictan por entidades como Cámara de Comercio, SENA, las Universidades, la gobernación y/o diferentes alcaldías del territorio nacional.

En cuanto al índice de desempleo de los economistas egresados en la Universidad de Nariño, para el periodo 2008-2013 fue del 7\%, y para Ortiz (2003) del 6\%, siendo valores inferiores al índice de desempleo nacional (11,1\%). Sin embargo, los resultados en Martínez (2010) presentan un nivel de desempleo del 14,3\% relativamente alto el cual afecta especialmente a la población joven y/o recién graduada. Lo citado anteriormente, permite inferir que si bien hay una relativa disminución del índice de desempleo en el periodo actual con respecto a periodos anteriores, se requiere generar acciones desde el Programa de Seguimiento a Egresados orientadas a reducir este indicador.

Tomando como referente el tiempo que demora un economista egresado de la Universidad de Nariño en ubicarse laboralmente, esta investigación aporta que 
en general oscila entre 6 y 18 meses teniendo en cuenta que la adhesión a un trabajo requiere el cumplimiento de ciertos requisitos limitando la posibilidad a un empleo estable por parte del recién egresado. Frente a esto, para el periodo 20082013 y Martínez (2010) se tiene como principal problema la falta de experiencia, sin embargo, es importante considerar la situación del departamento de Nariño y Colombia donde el mayor número de empresas son catalogadas como MIPYMES y cuya tendencia a fin de reducir costos es contratar bachilleres, técnicos y tecnólogos. Por otra parte, existen trabajos con labores que no son acordes a la formación del economista; destacando que ninguno de los profesionales encuestados señaló como motivo de desempleo aspectos tales como: bajos salarios, falta de conocimientos, salud, y la no búsqueda de empleo; e indicando disposición a vincularse laboralmente y ejercer su profesión. Es notoria la carencia de habilidades y competencias específicas requeridas por los empresarios al contratar un profesional, así como algunas limitantes entre ellas la temprana edad de los recién egresados siendo esta una de las principales dificultades para acceder a un cargo.

Asimismo, conseguir un empleo que garantice un bienestar económico estable es cada vez más difícil razón por lo cual muchos profesionales acceden a trabajos cuya característica principal es la informalidad. De acuerdo a esto, se obtiene que el $44 \%$ de los economistas desempleados están dispuestos a aceptar un trabajo si este les aporta seguridad social, el $22 \%$ si ofrece prestaciones sociales, y un $100 \%$ si el salario ofrecido está por encima del SMMV. En suma, frente a la actual situación de desempleo en el territorio nacional, hay egresados conscientes del complicado panorama laboral y optarían por empleos que incluso no reconozcan seguridad social y/o prestaciones sociales a fin de vincularse laboralmente.

\section{TRAYECTORIA EDUCATIVA}

Diversas instituciones de educación superior brindan alternativas de estudio orientadas a continuar el proceso de formación del egresado, como especializaciones, maestrías, doctorados, diplomados, etc. No obstante, a estas carreras pos graduales solo acceden aquellas personas interesadas en obtener un mayor grado de formación académica y que además, cuentan con recursos económicos para su financiación.

Para una mayor comprensión y caracterización del economista egresado de la Universidad de Nariño, se indagó sobre los estudios adelantados posterior a la obtención del título de pregrado: en general, el 52\% de los egresados continuó con su proceso de formación académica a través de especializaciones cursadas principalmente en el municipio de Pasto. En lo relacionado con estudios de maestrías, en el periodo 2008-2013 se observa un porcentaje representativo del 33\% que supera el 8\% y el 0\% indicado en Ortiz (2003) y Martínez (2010) respectivamente. A su vez, los profesionales con diplomados se encuentran especialmente en Martínez (2010) siendo el rango más característico. Se resalta en Ortiz (2003) un 16\% de economistas que han cursado carreras adicionales y otros cursos. Por último, solamente en el periodo 2008-2013 se tiene profesionales con estudios de doctorado en un $2 \%$ cuya ejecución se dio por fuera del país. 
Por otra parte, para el 48\% restante de la población sin estudios de posgrado el acceder nuevamente a la universidad se ha convertido prácticamente en un sueño, la existencia de factores de tipo socioeconómico como falta de recursos económicos, falta de tiempo, y la ausencia de programas específicos en la ciudad han dificultado este proceso. Es importante señalar que para un pequeño porcentaje la formación recibida en pregrado le ha sido suficiente para su desempeño laboral demostrando conformismo en este aspecto.

\section{PERCEPCIÓN DEL ECONOMISTA EN DIVERSOS TEMAS REFERENTES AL PROGRAMA DE ECONOMÍA}

La opinión del egresado es un factor de gran importancia al momento de generar e implementar estrategias con miras a un mayor fortalecimiento de los programas académicos. La aceptación o conformidad con la enseñanza del pregrado depende en gran medida de las posibilidades de inserción y estabilidad laboral. El determinar la relación existente entre la formación académica y el grado de productividad contribuye a generar acciones orientadas a desarrollar competencias y habilidades precisas en el futuro profesional para una adecuada inserción laboral.

En esta investigación se obtuvo que para un $61 \%$ de los economistas, la formación recibida no corresponde a las exigencias del mercado; frente a un 39\% que encuentra una relación marcada entre lo aprendido en la academia y las exigencias laborales. Los anteriores valores se asimilan a los de Ortiz (2003) y Martínez (2010) en los que se toma el aprendizaje como un indicador de productividad teniendo en cuenta el grado de satisfacción y el nivel de bienestar alcanzado. Es notorio como las condiciones actuales del mercado y la necesidad de una remuneración ha llevado a que muchos profesionales se desempeñen en campos diferentes a su formación académica.

Para solventar esta situación, desde la Universidad de Nariño se programan charlas, talleres, seminarios, foros, etc., orientados a que el egresado sea más competitivo. Sin embargo, la asistencia a dichos eventos es limitada por diversos factores como: desactualización de la base de datos, falta de tiempo, distancia hacia la universidad, bajo interés, entre otros.

Por otra parte, al considerar aspectos como pensum académico, infraestructura, profesores, misión, visión, evaluación y autorregulación, es importante hacer un análisis detallado de las fortalezas y debilidades existentes en el Programa de Economía, desde la percepción del egresado. Al sintetizar las diferentes opiniones relacionadas con el pensum académico, en general la calificación es buena, aunque muchos consideran que es regular y una minoría excelente y/o deficiente. En promedio, el pensum del programa según los egresados está entre bueno y regular sugiriendo la evaluación del mismo con criterios para el futuro profesional.

En cuanto a la infraestructura para el desarrollo del programa de Economía, se presenta una relativa mejora debido a la existencia de espacios adecuados dentro de 
la Universidad. Un pequeño grupo de profesionales considera que la infraestructura es regular e insiste en el mejoramiento y modernización de aulas y laboratorios.

Al valorar el papel desempeñado por los profesores se identificó un fuerte cambio en la percepción del profesional a lo largo de los años. En los datos de Ortiz 2003 y Martínez 2010 se tenía una imagen regular que oscilaba entre el $40 \%$ y $45 \%$, seguida de una opinión buena que fluctuaba entre el 47\% y 54\%. Al examinar el periodo 2008-2013 se observa una apreciación considerable, para un $4 \%$ y $72 \%$ respectivamente; el personal docente es excelente y bueno debido a la capacidad, compromiso y pedagogía utilizada. No obstante, para el 22\% es regular y asemejan esta calificación, a falencias académicas, metodología poco pertinente y falta de personal idóneo en temas que permitan un mejor desenvolvimiento en el ejercicio profesional.

Al considerar la percepción de la misión y visión del Programa de Economía, para el periodo 2008-2013 el 5\% de los egresados la considera excelente gracias a la contribución en la formación integral, el liderazgo y la autonomía de la persona. El 69\% tiene una apreciación aceptable por el trabajo continuo y la búsqueda de un desarrollo alternativo en beneficio de la región. El 25\% y 1\% de los encuestados la califican como regular y deficiente, respectivamente, argumentando problemas académicos, administrativos y financieros, que dan origen a una deficiente formación profesional.

Frente a los procesos de evaluación y autorregulación en el programa solamente el 4\% de los egresados está de acuerdo con que dicho proceso se cumple a cabalidad. El 59\% opina que estos procesos se desarrollan de manera parcial, argumentando que la Universidad maneja procesos de evaluación permanente, sin embargo, deja a un lado la implementación de acciones de mejoramiento. El 37\% restante expresa que se han identificado problemas específicos pero no se han desarrollado estrategias que ayuden al egresado en su crecimiento tanto profesional como integral.

En lo referente a la vinculación en comunidades y asociaciones de egresados, aspecto evaluado para evidenciar la contribución al bienestar social y el mejoramiento regional sin olvidar las distinciones y/o reconocimientos alcanzados por la labor, compromiso, dedicación y entrega en las funciones que desempeña el profesional; para el periodo 2008-2013 se tiene que un pequeño porcentaje de los egresados es miembro activo de alguna comunidad: el 4,9\% en una agremiación del sector productivo, el 8,9\% en una asociación de profesionales, el 5,7\% en una asociación científica y el 13\% en una comunidad académica. Un 20,3\% ha recibido distinciones y/o reconocimientos significativos por su desempeño en la disciplina, profesión, ocupación u oficio correspondiente. Asimismo, frente a este aspecto en Martínez 2010 se tiene que el 22\% ha participado de gremios y/o asociaciones en donde la generación 1979-1988 es la de mayor participación. Lo anterior refleja una situación positiva demostrando como los economistas sobresalen en sus labores, trayendo consigo prestigio y credibilidad a la Universidad de Nariño. 
De acuerdo a lo anterior, la formación académica y las distinciones recibidas en la universidad y en la vida laboral, permiten un mayor desenvolvimiento profesional al igual que una mayor eficiencia en el cargo desempeñado. Conjuntamente, se precisa una buena formación integral tomando como base la participación de la academia en la formación de personas con sentido ético y posibilidades de participación en el desarrollo regional.

\section{PROPUESTA DE SEGUIMIENTO A LOS EGRESADOS DEL PROGRAMA DE ECONOMÍA DE LA UNIVERSIDAD DE NARIÑO}

La evaluación de la situación real del egresado constituye una herramienta esencial a la hora de identificar la pertinencia del Programa frente a las necesidades del entorno y las exigencias de la sociedad. A partir del análisis económico y social de los egresados se tienen algunos insumos requeridos para formular e implementar estrategias y acciones orientadas a solventar carencias y debilidades de tipo académico existentes en el programa de Economía.

En atención a lo anterior, se estudió las diversas observaciones y recomendaciones hechas por parte de los egresados para mejorar la calidad del programa de Economía, entre ellas: mayor crecimiento y oportunidad dentro de la Universidad a los nuevos profesionales, fortalecimiento de los estímulos educativos con becas de posgrado, intercambios académicos y prácticas empresariales; una mayor articulación con las empresas y gremios de la región, renovación del pensum académico de acuerdo a las necesidades de la región, y el mejoramiento de los convenios empresariales.

Asimismo, para el fortalecimiento en la formación académica del estudiante y un mejor desempeño profesional, es fundamental la contribución de los directivos y demás entes encargados. Al respecto, se observa que existe apoyo a la iniciativa de implementar un Programa de seguimiento a Egresados, en razón de que al momento el Programa de Economía no cuenta con un programa de egresados y con talento humano para su seguimiento, destacando además, que el programa de seguimiento a egresados es evaluado como una característica de calidad por parte del Consejo Nacional de Acreditación, CNA. Igualmente, se identifica la desmotivación de los egresados para asistir a eventos programados por la Facultad de Ciencias Económicas y Administrativas FACEA, argumentando diferentes razones, entre ellas, las mencionadas anteriormente.

En razón a lo anteriormente citado, y como medio para la búsqueda de posibles salidas a estos inconvenientes a partir de la investigación y de las observaciones y recomendaciones realizadas por los egresados y directivos, se desarrolló la matriz DOFA (análisis de debilidades, oportunidades, fortalezas y amenazas) acompañada de estrategias de acción, a fin de señalar las pautas para crear y constituir un programa continuo de seguimiento a egresados que beneficie a los profesionales del programa de Economía en la Universidad de Nariño. 


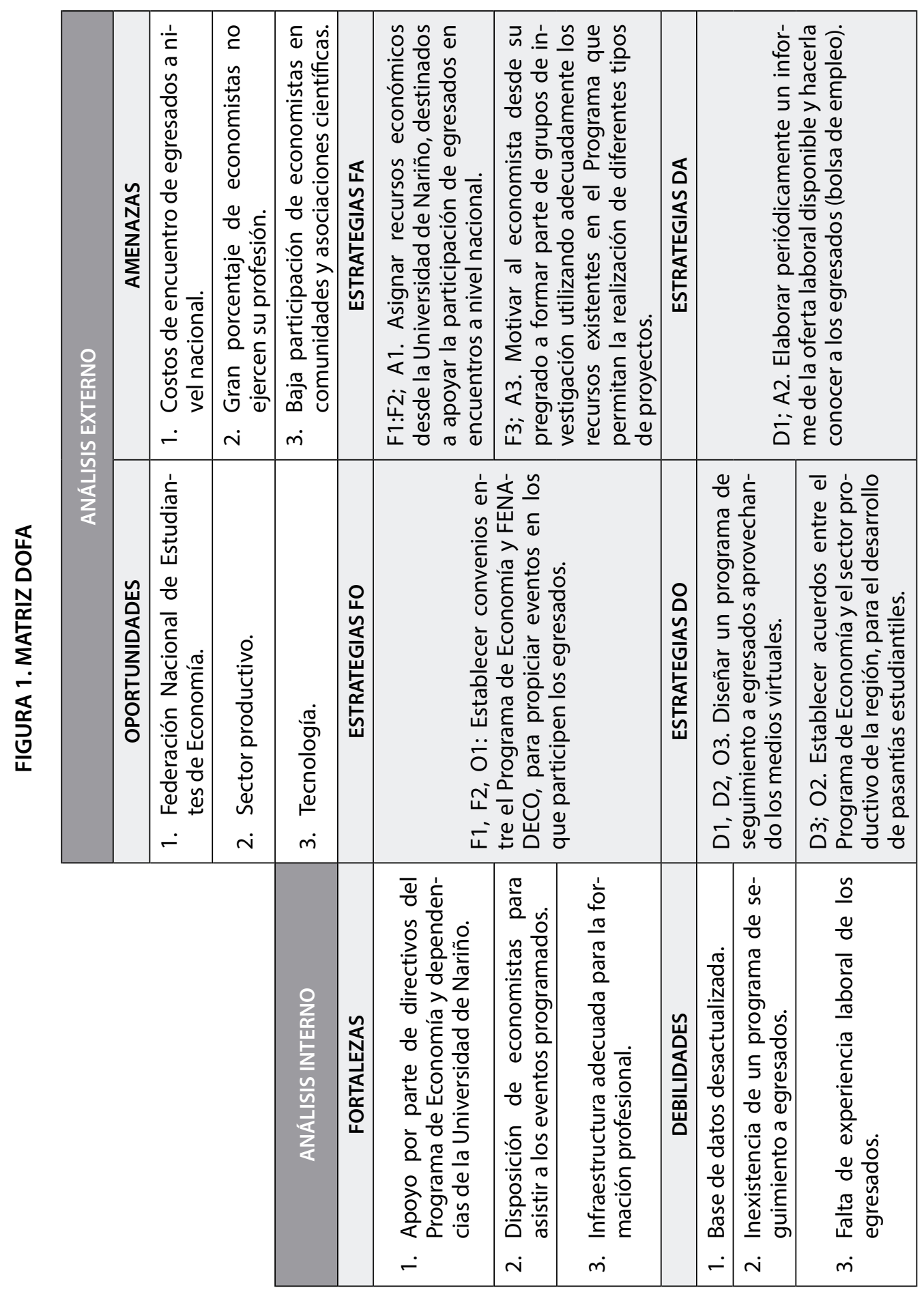




\begin{tabular}{|c|c|c|c|c|c|c|c|}
\hline 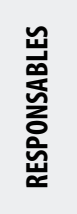 & \multicolumn{3}{|c|}{ 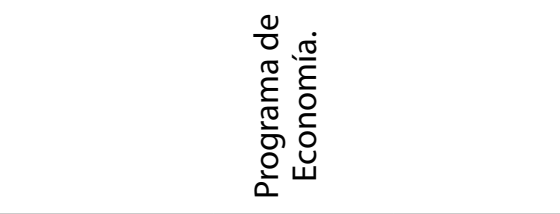 } & \multicolumn{4}{|c|}{ 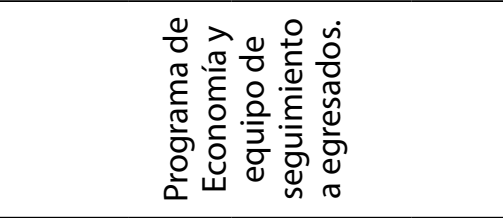 } \\
\hline 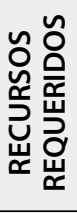 & \multicolumn{3}{|c|}{ 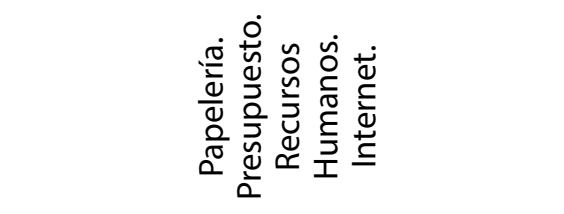 } & \multicolumn{4}{|c|}{ 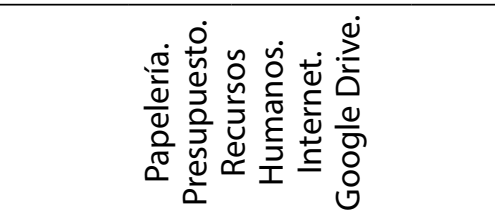 } \\
\hline 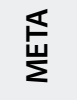 & ઠे & $\stackrel{\circ}{\circ}$ & ઠे & ઠे & §̊ & ठి & ஓ̊ \\
\hline 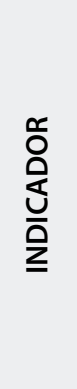 & 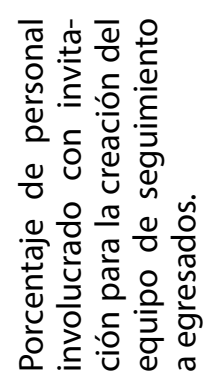 & 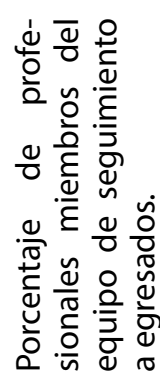 & 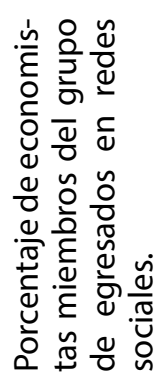 & 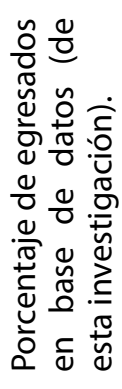 & 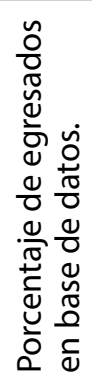 & 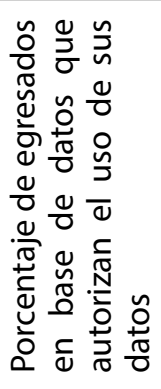 & 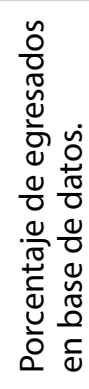 \\
\hline 岂 & 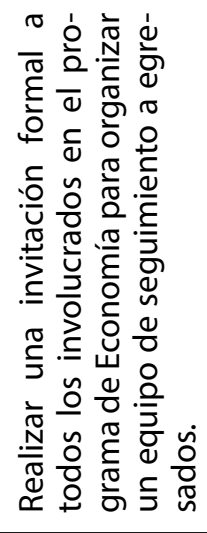 & 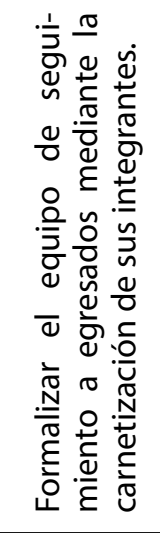 & 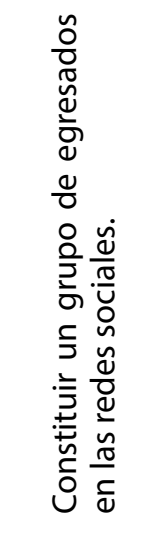 & 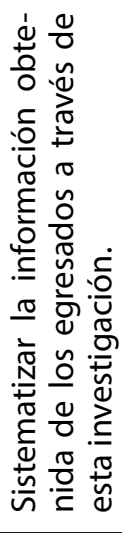 & 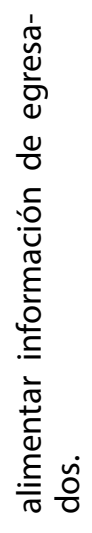 & 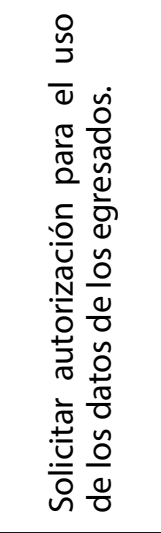 & 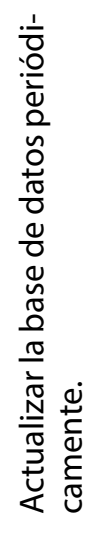 \\
\hline 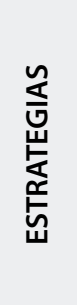 & \multicolumn{3}{|c|}{ 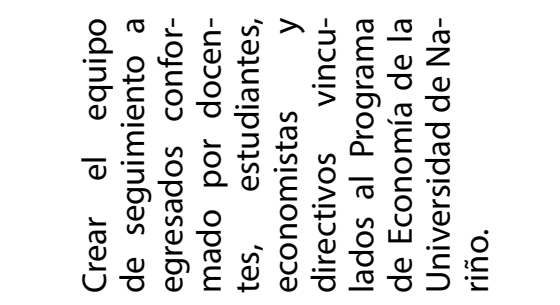 } & \multicolumn{4}{|c|}{ 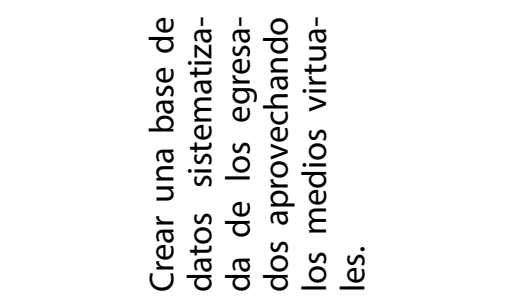 } \\
\hline$z$ & \multicolumn{3}{|c|}{-} & \multicolumn{4}{|c|}{$\sim$} \\
\hline
\end{tabular}




\begin{tabular}{|c|c|c|c|c|c|c|c|}
\hline 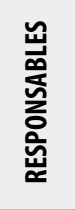 & \multicolumn{2}{|c|}{ 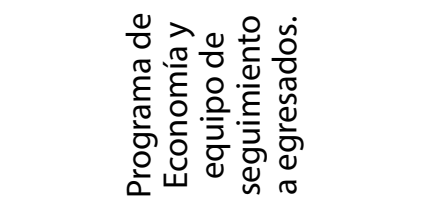 } & \multicolumn{2}{|c|}{ 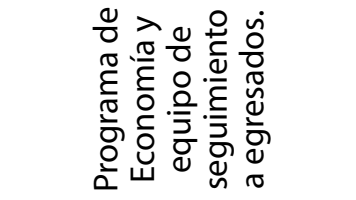 } & \multicolumn{3}{|c|}{ 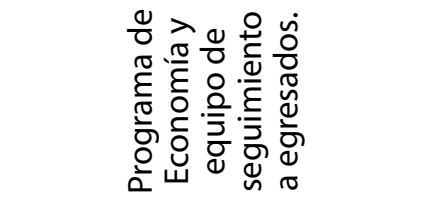 } \\
\hline 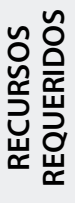 & \multicolumn{2}{|c|}{ 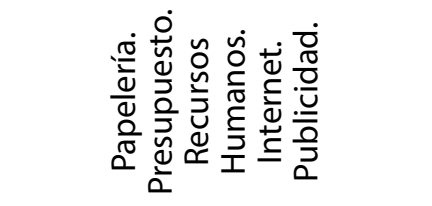 } & \multicolumn{2}{|c|}{ 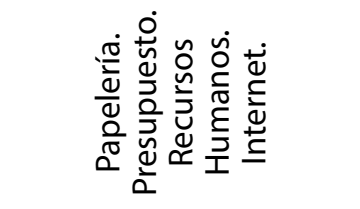 } & \multicolumn{3}{|c|}{ 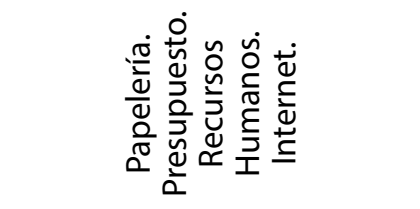 } \\
\hline$\underset{\Sigma}{\stackrel{\rightleftarrows}{\rightleftarrows}}$ & ठ̊̊ & ઠे & ᄋ̊̀ & ஓे & ஓे & ঃ̊ & ○े \\
\hline 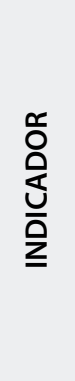 & 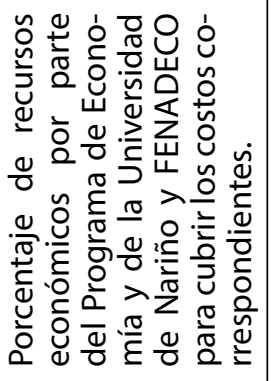 & 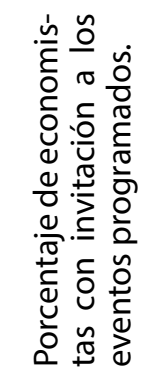 & 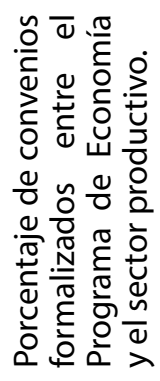 & 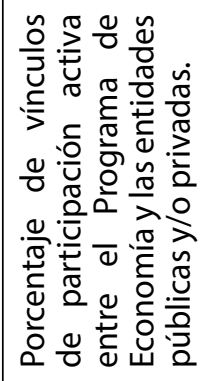 & 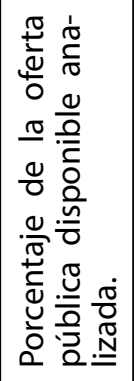 & 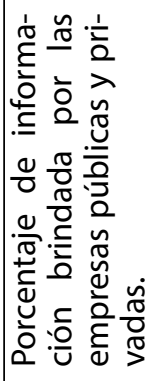 & 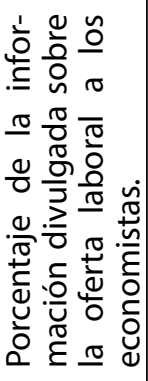 \\
\hline 岂 & 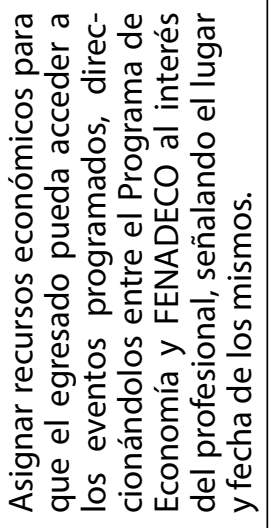 & 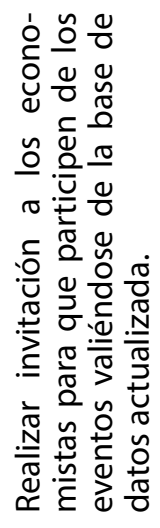 & 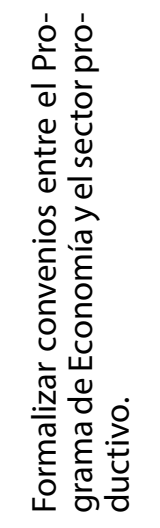 & 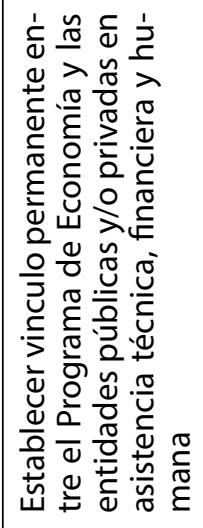 & 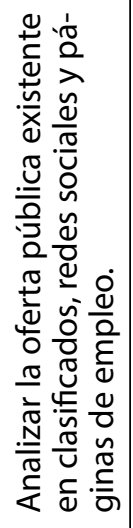 & 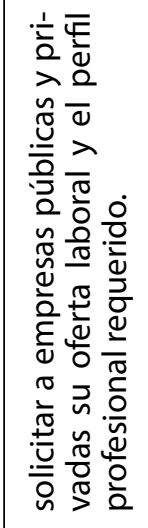 & 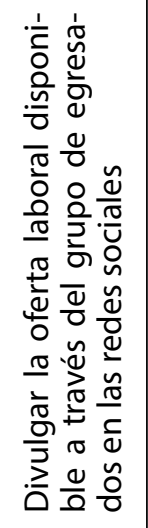 \\
\hline 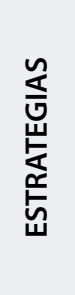 & \multicolumn{2}{|c|}{ 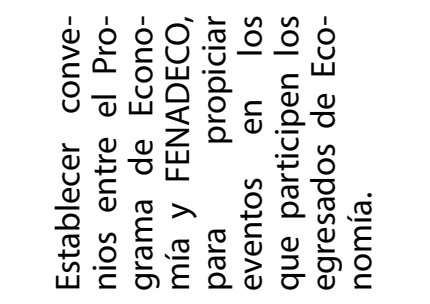 } & \multicolumn{2}{|c|}{ 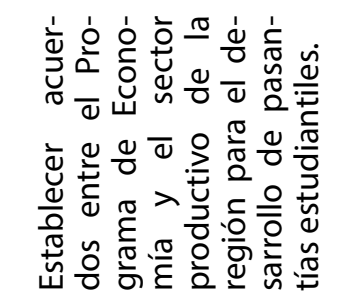 } & \multicolumn{3}{|c|}{ 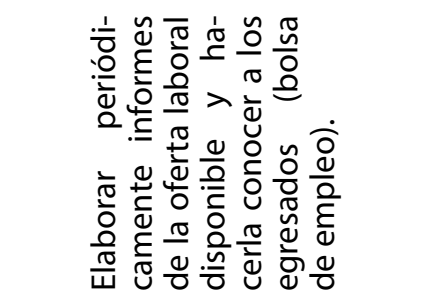 } \\
\hline $\mathbf{z}$ & \multicolumn{2}{|l|}{$m$} & \multicolumn{2}{|r|}{ } & \multicolumn{3}{|c|}{ in } \\
\hline
\end{tabular}




\section{CONCLUSIONES}

De acuerdo a los datos encontrados en esta investigación y a las investigaciones desarrolladas por los economistas Robert Wilson Ortiz y Pablo Sebastián Martínez, se tiene:

- El porcentaje de hombres y mujeres egresados en el programa de Economía de la Universidad de Nariño desde la primera promoción hasta el año 2013 ha sido equitativo. Se encontró primordialmente jóvenes solteros, seguido de personas casadas y en unión libre, ubicados en los estratos medios de la sociedad y localizados en su mayoría en el departamento de Nariño, como punto principal el municipio de Pasto. Se resalta un número considerable de egresados sin casa propia, aunque cuentan con los servicios necesarios en sus viviendas, lo cual les ha permitido vivir dignamente.

- Existe correspondencia entre los economistas empleados en empresas públicas y en entidades privadas. Los egresados más jóvenes, principalmente, están vinculados laboralmente a término fijo y los que gozan de una mayor experiencia en periodo indefinido. Además, la mayoría de egresados está afiliado al régimen contributivo, seguido del régimen subsidiado, y medicina pre-pagada. Lo anterior posibilita al egresado, acceder a las atenciones que le garanticen su recuperación y protección personal.

- La mayor parte de los economistas laboran en el sector terciario, especialmente en áreas financieras, de talento humano, comerciales y de producción, con ingresos que oscilan entre 1 y 4 SMMV. Se destaca que muchos se han vinculado al mercado laboral, por recomendaciones de terceras personas.

- Muy pocos economistas han optado por constituir un negocio propio, siendo trascendental dentro del programa de Economía fortalecer las áreas de emprendimiento, puesto que la existencia de diversos factores como la falta de información, la distancia, y/o poco interés en asistir a las capacitaciones que se dictan por parte de la Cámara de Comercio, el SENA, las universidades, las gobernaciones y/o diferentes alcaldías del territorio nacional, ha llevado a que cada día sean mayor el número de empleados que el número de empleadores.

- Los economistas independientes en su mayoría son microempresarios, con empresas o negocios vinculados, principalmente al sector terciario en actividades de: servicios comunitarios, sociales y/o personales, de servicios sociales y de salud, y comercio al por menor. Asimismo, sus ganancias oscilan entre 1 y 4 SMMV. Por último, el 77\% de los egresados, no tiene afiliación a salud, prestaciones sociales, pensión y riesgos profesionales, puesto que según ellos, al no emplear personal de manera permanente no hay una obligación laboral inmediata, llevándolos a una vinculación al régimen subsidiado.

- Al comparar los bajos índices de desempleo en Ortiz 2003 (6\%) y del periodo 2008-2013 (7\%); con el índice nacional (11,1\%), se tiene una oportunidad laboral por la existencia de profesionales trabajando en una empresa o negocio propio. Se resalta en Martínez (2010) un nivel de desempleo relativamente alto, expresado en un 14,3\% que afecta, en especial a la población joven y/o recién graduada. 
- El tiempo que busca empleo el economista de la Universidad de Nariño generalmente oscila entre los 6 a 18 meses; teniendo como principal obstáculo la falta de experiencia y la falta de vacantes.

- La mayor parte de economistas que continuaron con estudios posteriores los realizaron principalmente en Pasto, en especializaciones, maestrías y/o diplomados; solamente en el periodo 2008-2013, se tiene profesionales con estudios de doctorado representados en un 2\%, cuya ejecución se dio por fuera del país. Por otra parte, existe un gran porcentaje que no siguió estudios de posgrado debido la falta de recursos económicos, la falta de tiempo y la inexistencia en la ciudad del programa requerido, y buscan el apoyo de los diferentes entes existentes en el país para poder continuar con su formación profesional.

- Para el 61\% de los egresados la formación recibida no corresponde a las exigencias del mercado, frente a un 39\% que encuentra una relación marcada entre lo aprendido en la academia y las exigencias laborales. Es notorio, como las condiciones actuales del mercado y la necesidad de una remuneración económica ha llevado a muchos profesionales a trabajar en campos diferentes a su formación académica.

- Al calificar al programa de Economía, sobresale como fortaleza la infraestructura, no obstante es preciso señalar que el pensum, el personal docente, la misión, la visión, la evaluación y la autorregulación, son calificados como buenos, es decir, la percepción del egresado se mantiene en términos intermedios. Existe una necesidad de mejora en diversos temas como: la implementación de materias y la estructuración del pensum, el mejoramiento de la metodología aplicada, con fundamentos en las necesidades de la región, modernización de aulas y laboratorios, y fortalecimiento en las técnicas de evaluación y autorregulación, lo anterior con el fin de incrementar la imagen que proyecta la universidad.

- De acuerdo a las observaciones de los egresados para mejorar la calidad educativa, es importante destacar: un mayor crecimiento y oportunidades dentro de la Universidad a los nuevos profesionales, el fortalecimiento de los estímulos educativos como becas de posgrado, intercambios académicos, prácticas empresariales, y una mayor articulación con las empresas y gremios de la región, para que los egresados puedan adquirir experiencia laboral.

- Los directivos y entes, encargados en la Universidad de Nariño, afirman que no existe un programa y un equipo de seguimiento continuo al egresado, siendo de suma importancia para los procesos de acreditación en alta calidad, además de permitir conocer la situación real del profesional.

- Con la realización de un diagnóstico, fue posible establecer los factores claves que intervienen en el proceso de seguimiento de los egresados, de esta manera se establecieron diversas estrategias que permiten tener en cuenta a los profesionales en los procesos que se llevan a cabo dentro de la Universidad.

- Es importante conocer y aprovechar las fortalezas y oportunidades para eliminar las debilidades y contrarrestar las amenazas existentes en la formación acadé- 
mica, con esta herramienta es posible dilucidar un mejor desarrollo profesional de los economistas y futuros economistas de la Universidad de Nariño.

- El programa de seguimiento debe contar con una base de datos actualizada y un equipo de trabajo idóneo, para conocer el estado actual de los economistas, dándole continuidad a los procesos y estrategias adelantadas.

- Un programa de calidad lleva implícita la responsabilidad de estar en continuo contacto con sus egresados, conocer su desempeño profesional e identificar si la formación brindada es acorde a las exigencias del mercado, por lo que la inserción laboral de los egresados es parte de su compromiso social con el desarrollo de la región.

\section{REFERENCIAS}

1. CONSEJO NACIONAL DE ACREDITACIÓN (2013). Lineamientos para la acreditación de programas de pregrado. Bogotá.

2. CONSTITUCIÓN NACIONAL (1991). LEY 30 DE 1992: Por lo cual se otorga el servicio público de la educación superior.

3. LEY 115 DE 1994. Por la cual se expide la Ley General de la Educación. Febrero 8.

4. LEY 1188 de 2008.

5. MARTínEZ, Pablo Sebastián (2010). Caracterización y aporte al desarrollo regional de los economistas egresados del programa de Economía de la Universidad de Nariño sede Pasto, San Juan de Pasto. 2010.

6. MINISTERIO DE EDUCACIÓN NACIONAL. Decreto 1295 de 2010. Bogotá. Abril 20.

7. ORDÓÑEZ, Luis Aurelio (2013). En: Revista TENDENCIAS, de la Facultad de Ciencias Económicas y Administrativas. Universidad de Nariño. Volumen XIV, No. 1. Editorial Unariño.

8. ORTIZ, Robert Wilson (2003) Caracterización de los Egresados del Programa de Economía de la Universidad de Nariño. San Juan de Pasto.

9. PORTAFOLIO (2013). www.portafolio.co/economia/desempleocolombia.

10. RED GRADUA2 (2006). Manual de instrumentos y recomendaciones sobre el seguimiento de egresados. México.

11. UNIVERSIDAD AUTÓNOMA DEL ESTADO DE MORELOS. Programa de seguimiento a egresados (2007). México.

12. UNIVERSIDAD DE COLIMA (2009). Programa institucional de seguimiento de egresados. México.

13. UNIVERSIDAD DEL SABES (2011). Evaluación de perfil de egreso y seguimiento de egresados del bachillerato SABES. México. 